a quadratic equation having integral coefficients. Carmichael has given a different and more exhaustive treatment of these numbers in the Annals of Mathematics, 1913. In the present paper Mr. Pierce obtains somewhat similar results for numbers given by the forms $\prod_{i=1}^{n}\left(1 \pm \alpha_{i}{ }^{m}\right)$, where the $\alpha_{i}$ denote algebraic integers defined as the roots of an $n$th degree equation. The forms of the factors of $\prod_{i=1}^{n}\left(1-\alpha_{i}{ }^{m}\right)$ are determined by use of algebraic number theory, and this perhaps constitutes the most novel result of the work.

6. Lucas has developed the theory of the prime divisors of the functions $U_{n}=\left(a^{n}-b^{n}\right) /(a-b)$ and $V_{n}=a^{n}+b^{n}$, where $a$ and $b$ are the roots of a quadratic equation (American Journal of Mathematics, volume 1, page 184). Connected with these functions are certain binary forms of degree equal to one half the totient of $n$, the divisors of which Professor Lehmer has shown to be of the form $2 n x \pm 1$. Combining this result with certain results of Mr. Pierce, Professor Lehmer has also obtained a series of numbers the prime factors of which must belong to two such forms, thus restricting notably the character of their divisors.

7. Professor Haskell shows that the condition that a rational fraction whose denominator is the $n$th power of a quadratic should be rationally integrable, is that the numerator shall be of degree $2(n-1)$ and that it shall be apolar to the $(n-1)$ st power of the quadratic factor of the denominator.

Thomas Buck, Secretary of the Section.

\title{
TRANSFORMATION THEOREMS IN THE THEORY OF THE LINEAR VECTOR FUNCTION.
}

\author{
BY DR. VINCENT C. POOR.
}

(Read before the American Mathematical Society, December 31, 1915.)

SrNCE the memorable work of Grassmann (1844), the study of the linear transformation has taken various forms, among which are the quaternions of Hamilton, the matrices of Cayley, 
the dyadics of Gibbs, and the homography as treated by BuraliForti and Marcolongo. The notation here followed is that of the book on "Transformations Linéaires" by the last mentioned authors. Reference to the French (1912) edition of this work will be briefly made by the letters B. M. with the section and number following.

The homography is defined by Burali-Forti and Marcolongo* as any linear operator which transforms vectors into vectors. One of the simplest examples of a homography is $\boldsymbol{u} \wedge$, the axial homography, $\dagger$ which transforms all vectors into vectors perpendicular to the vector $\boldsymbol{u}$.

Another concept of fundamental importance in advanced vector analysis is the Grassmann point derivative. If $M$ and $P$ are any two points of space, then $M-P$ is the vector represented by the line segment directed from $P$ to $M . \ddagger$ If we use $\boldsymbol{u}$ for the difference between any two points of space, and if $f(P)$ is a function depending on the point $P$, the differential of $f$, written $d f$, $\delta f$, etc., may be defined briefly by the equation

$$
d f=\lim _{h \doteq 0} \frac{f(P+h \boldsymbol{u})-f(P)}{h} .
$$

From this it follows that

$$
d P=\boldsymbol{u}
$$

or $d P$ is any arbitrary vector. The definition of the point derivative $d f / d P$, following the Leibnitz notation, may now be expressed by the equation

$$
\delta f=\frac{d f}{d P} \delta P, \S
$$

i. e., $d f / d P$ is an operator on any arbitrary vector $\delta P$ which transforms that vector into $\delta f$. It may be shown that $d f / d P$ is a linear operator. In no sense is $d f / d P$ to be regarded as a quotient. When the operator $d f / d P$ transforms the operand, a vector, into a vector, the point derivative furnishes another example of a homography.

* B. M., 1.

$\dagger$ The cross $\times$ and the inverted $\vee(\wedge$, read "vec"), standing between two vectors, indicate the scalar and vector products, respectively.

$\ddagger$ The vector appears as a particular case of the Grassmann "first formation."

$\S$ B. M., p. 60,5 . 
If $\boldsymbol{u}$ and $\boldsymbol{v}$ are two arbitrary vectors, then the vector of a homography $\alpha$, designated by $V \alpha$, may be defined as a vector such that

$$
2 V \alpha \times \boldsymbol{u} \wedge \boldsymbol{v}=\boldsymbol{v} \times \alpha \boldsymbol{u}-\boldsymbol{u} \times \alpha \boldsymbol{v} . *
$$

It is easy to show that

$$
\frac{d(\alpha \boldsymbol{u})}{d P} \boldsymbol{x}=\alpha \frac{d \boldsymbol{u}}{d P} \boldsymbol{x}+\left(\frac{d \alpha}{d P} \boldsymbol{x}\right) \boldsymbol{u}, \dagger
$$

where $\alpha$ is a homography and $\boldsymbol{u}$ and $\boldsymbol{x}$ are vectors. Transposing the first term of the right member, we have

$$
\left(\frac{d \alpha}{d P} \boldsymbol{x}\right) \boldsymbol{u}=\left[\frac{d(\alpha \boldsymbol{u})}{d P}-\alpha \frac{d \boldsymbol{u}}{d P}\right] \boldsymbol{x} .
$$

For the brackets in the right member, $S_{P}(\alpha, \boldsymbol{u})$ will be written. This binary operator is evidently a homography.

The rotational of a homography (written " $\operatorname{Rot} \alpha$ "), may be briefly defined as a homography such that

$$
(\operatorname{Rot} \alpha) \boldsymbol{u}=2 V S(\alpha, \boldsymbol{u}) .
$$

The theorems of the present paper are linear transformation theorems which involve the homography, in general, as a function of two points of space. The letter $P$ will be used throughout as the point of integration. It will be understood that the surface $\sigma$ bounds the region $\tau$ and that $n$ is a unit normal at a point $P$ of the surface $\sigma$ with its positive sense towards the interior of $\sigma$.

Theorem 1. If $\alpha$ is a homography symmetric in $P$ and $M$, such that

$$
\frac{d \alpha}{d M}=-\frac{d \alpha}{d P}
$$

and if $\boldsymbol{u}$ is independent of $M$, then

$$
2 \int V \frac{d(\alpha \boldsymbol{u})}{d M} d \tau=\int \boldsymbol{n} \wedge \alpha \boldsymbol{u} d \sigma+2 \int V\left(\alpha \frac{d \boldsymbol{u}}{d P}\right) d \tau .
$$

For, taking $x$ as a function of $M$ alone, and applying (1) and the hypotheses of the theorem, we find that

$$
\frac{d(\alpha \boldsymbol{u})}{d M} \boldsymbol{x}=\left(\frac{d \alpha}{d M} \boldsymbol{x}\right) \boldsymbol{u}=-\left(\frac{d(\alpha \boldsymbol{u})}{d P}-\alpha \frac{d \boldsymbol{u}}{d P}\right) \boldsymbol{x} .
$$

* B. M., 8 [2].

† B. M., 36 [1]. 
Remembering the definition of $S$ this result may be written

$$
\frac{d(\alpha \boldsymbol{u})}{d M}=-S_{P}(\alpha, \boldsymbol{u})
$$

or by using (2), the definition for the rotational of a homography, we have

$$
2 V \frac{d(\alpha \boldsymbol{u})}{d M}=-2 V S_{P}(\alpha, \boldsymbol{u})=-\left(\operatorname{Rot}_{P} \alpha\right) \boldsymbol{u} .
$$

By substituting this result in the known transformation formula

$$
\int\left(\operatorname{Rot}_{P} \alpha\right) \mathbf{u} d \tau=-\int \boldsymbol{n} \wedge \alpha \mathbf{u} d \sigma-2 \int V\left(\alpha \frac{d \mathbf{u}}{d P}\right) d \tau,{ }^{*}
$$

the theorem will be obtained. This theorem may be put into a slightly different form if we introduce the definition for the rotational $\dagger$ of a vector, written " rot," which is expressed by the equation

$$
\operatorname{rot}_{P} \boldsymbol{u}=2 V \frac{d \mathbf{u}}{d P} .
$$

The theorem will then read

$$
\int \operatorname{rot}_{M} \alpha \boldsymbol{u} d \tau=\int \boldsymbol{n} \wedge \alpha \boldsymbol{u} d \sigma+2 \int V\left(\alpha \frac{d \boldsymbol{u}}{d P}\right) d \tau .
$$

In expressing the next theorem we will need the conjugate of a homography $K \alpha$, the first invariant of a homography $I_{1} \alpha$, and the gradient of a homography $\operatorname{grad} \alpha$, of which the gradient of a scalar is a special case. The Maxwell divergence of a vector, which arises, is to be found in any book on the elements of vector analysis. The conjugate of a homography may be defined by the equation

$$
K \alpha=\alpha-2 V \alpha \wedge,
$$

which is again a homography. The first invariant of a homography $\alpha$ is a scalar, which, for any three arbitrary vectors $\boldsymbol{u}, \boldsymbol{v}, \boldsymbol{w}$, satisfies the following relation:

$\boldsymbol{u} \wedge \boldsymbol{v} \times \boldsymbol{w} \cdot I_{1} \alpha=\boldsymbol{v} \wedge \boldsymbol{w} \times \alpha \boldsymbol{u}+\boldsymbol{w} \wedge \boldsymbol{u} \times \alpha \boldsymbol{v}+\boldsymbol{u} \wedge \boldsymbol{v} \times \alpha \boldsymbol{w}$.

* B. M., 56 [3], second.

$\dagger$ The rotational of a vector is identical with the Maxwell curl, or the Gibbs $\nabla \times$. 
The gradient of a homography $\alpha$, function of the point $P$, is a vector such that, for any arbitrary vector $\boldsymbol{u}$,

$$
\operatorname{grad}_{P} \alpha \times \boldsymbol{u}=I_{1} S_{P}(K \alpha, \boldsymbol{u}) \text {.* }
$$

Theorem 2. If $\alpha$ is a homography function of $P$ and $M$, such that

$$
\frac{d \alpha}{d M}=-\frac{d \alpha}{d P}
$$

and if $\mathbf{u}$ is a function independent of $M$, then

$$
\int \operatorname{div}_{M} \alpha \boldsymbol{u} d \tau=\int \boldsymbol{n} \times \alpha \boldsymbol{u} d \sigma+\int I_{1}\left(\frac{d \mathbf{u}}{d P} \alpha\right) d \tau .
$$

The proof of this theorem follows easily from the following known theorems, namely:

$$
\operatorname{div}_{M} \alpha \boldsymbol{u}=\boldsymbol{u} \times \operatorname{grad}_{M} K \alpha+I_{1}\left(\alpha \frac{d \boldsymbol{u}}{d M}\right), \dagger
$$

which reduces, since $\boldsymbol{u}$ is independent of $M$, to

and

$$
\operatorname{div}_{M} \alpha \boldsymbol{u}=-\boldsymbol{u} \times \operatorname{grad}_{P} K \alpha,
$$

$$
\int \operatorname{grad}_{P} \alpha \times \boldsymbol{u} d \tau=-\int \boldsymbol{u} \times \alpha \boldsymbol{n} d \sigma-\int I_{1}\left(\frac{d \boldsymbol{u}}{d P} K \alpha\right) d \tau, \ddagger
$$

which may be written

$$
\int \boldsymbol{u} \times \operatorname{grad}_{P} K \alpha d \tau=-\int \boldsymbol{u} \times K \alpha \boldsymbol{n} d \sigma-\int I_{1}\left(\frac{d \boldsymbol{u}}{d P} \alpha\right) d \tau .
$$

The substitution of $-\operatorname{div}_{M} \boldsymbol{u}$ for its equal in the left member of the last equation and the application of the commutation theorem

$$
\boldsymbol{n} \times \alpha \boldsymbol{u}=\boldsymbol{u} \times K \alpha \boldsymbol{n}
$$

lead to the theorem as stated.

The dyad $H(\boldsymbol{u}, \boldsymbol{v})$, another binary operator, is defined by the equation

$$
H(\boldsymbol{u}, \boldsymbol{v}) x=\boldsymbol{u} \times \boldsymbol{x} \cdot \boldsymbol{v} .
$$

The dyad is thus seen to be a homography. We may now express

* B. M., 8 and 37 [3].

† B. M., 41 [3], second.

$\$$ B. M., 56 [2], first. 
Theorem 3. If $\alpha$ is a homography, symmetric in $P$ and $M$, such that

$$
\frac{d \alpha}{d \bar{M}}=-\frac{d \alpha}{d P},
$$

and if $\boldsymbol{u}$ is independent of $M$, then

$$
\int \frac{d(\alpha \boldsymbol{u})}{d M} d \tau=\int H(\boldsymbol{n}, \alpha \boldsymbol{u}) d \sigma+\int \alpha \frac{d \boldsymbol{u}}{d P} d \tau .
$$

To demonstrate this theorem we may first observe that

$$
\int\left(\operatorname{div}_{P} \boldsymbol{v}\right) \cdot \boldsymbol{u} d \tau=-\int \boldsymbol{v} \times \boldsymbol{n} \cdot \boldsymbol{u} d \sigma-\int \frac{d \boldsymbol{u}}{d P} \boldsymbol{v} d \tau .^{*}
$$

Replacing $\boldsymbol{v}$ by $\boldsymbol{x}$ and $\boldsymbol{u}$ by the vector $\alpha \boldsymbol{u}$, this formula will become

$$
\int\left(\operatorname{div}_{P} \boldsymbol{x}\right) \cdot \alpha \boldsymbol{u} d \tau=-\int \boldsymbol{n} \times \boldsymbol{x} \cdot \alpha \boldsymbol{u} d \sigma-\int \frac{d(\alpha \boldsymbol{u})}{d P} d \tau .
$$

Under the assumption that $x$ is independent of $P$ this readily reduces to

$$
\int S_{P}(\alpha, \boldsymbol{u}) d \tau=-\int H(\boldsymbol{n}, \alpha \boldsymbol{u}) d \sigma-\int \alpha \frac{d \boldsymbol{u}}{d P} d \tau .
$$

With the same restriction on the $x$ we have

$$
\frac{d(\alpha \boldsymbol{u})}{d} \bar{M}=\left(\frac{d \alpha}{d M} \boldsymbol{x}\right) \boldsymbol{u}=-S_{P}(\alpha, \boldsymbol{u}) x .
$$

From these considerations the theorem will be evident. When the surface integral is identically zero, we have the useful corollary

$$
\int \frac{d(\alpha \boldsymbol{u})}{d M} d \tau=\int \alpha \frac{d \boldsymbol{u}}{d P} d \tau
$$

This situation could well happen in the case of an infinite region.

THeOREM 4. If $\alpha$ is a homography function of $P$ and $M$ and if $\boldsymbol{u}$ is independent of $M$, and $\boldsymbol{x}$ independent of $P$, then

$$
\int \frac{d\left(\alpha \frac{d \mathbf{u}}{d P}\right)}{d M} x d \tau=\int\left(\frac{d \alpha}{d M} \boldsymbol{x}\right) \frac{d \boldsymbol{u}}{d P} d \tau .
$$

\footnotetext{
* B. M., 56 [3], first.
} 
This theorem is demonstrated as soon as the integrands are shown to be equal. Introducing the vector $\boldsymbol{y}$, a function of $M$ alone, then the expression

$$
\frac{d\left(\alpha \frac{d \boldsymbol{u}}{d P} \boldsymbol{y}\right)}{d M} \boldsymbol{x}
$$

may be written in two different forms, ${ }^{*}$ namely

and

$$
\alpha \frac{d\left(\frac{d \mathbf{u}}{d P} \boldsymbol{y}\right)}{d M} \boldsymbol{x}+\left(\frac{d \alpha}{d M} \boldsymbol{x}\right) \frac{d \boldsymbol{u}}{d P} \boldsymbol{y}
$$

$$
\alpha \frac{d \mathbf{u}}{d P} \frac{d \boldsymbol{y}}{d M} \boldsymbol{x}+\frac{d\left(\alpha \frac{d \mathbf{u}}{d P}\right)}{d M} \boldsymbol{y} .
$$

But by the hypotheses of the theorem, the first terms of these expressions may be seen to be equal. The last two are then equal and the theorem follows.

Theorem 5. If $\alpha$ is a homography symmetric in $P$ and $M$ and if $\boldsymbol{u}$ is a function independent of $M$, then

$$
\begin{aligned}
\int \Delta_{M}{ }^{\prime}(\alpha \boldsymbol{u}) d \tau=-\int\left\{\left(\frac{d \alpha}{d P} \boldsymbol{n}\right) \boldsymbol{u}-\left(\alpha \frac{d \boldsymbol{u}}{d P}\right)\right. & \boldsymbol{n}\} d \sigma \\
& +\int \alpha\left(\Delta_{P}{ }^{\prime} \boldsymbol{u}\right) d \tau .
\end{aligned}
$$

The new symbols involved in this theorem may be defined as follows:

$$
\begin{aligned}
\Delta_{P}^{\prime} \boldsymbol{u} & =\operatorname{grad} \frac{d \boldsymbol{u}}{d P}, \\
\left(\Delta_{P} \alpha\right) \boldsymbol{u} & =\operatorname{grad}\left\{\frac{d(\alpha \boldsymbol{u})}{d P}-2 \alpha \frac{d \boldsymbol{u}}{d P}\right\}+\alpha \operatorname{grad} \frac{d \boldsymbol{u}}{d P} .
\end{aligned}
$$

From these definitions it follows at once that

$$
\Delta_{P}{ }^{\prime}(\alpha \boldsymbol{u})=\left(\Delta_{P} \alpha\right) \boldsymbol{u}-\alpha\left(\Delta_{P}{ }^{\prime} \boldsymbol{u}\right)+2 \operatorname{grad}_{P}\left(\alpha \frac{d \boldsymbol{u}}{d P}\right) .^{\dagger}
$$

* Apply equation (1).

$\dagger$ B. M., 50 [3]. The parentheses may be omitted, since $\Delta$ operates on homographies only, while $\Delta^{\prime}$ operates only on vectors. 
Applying our hypotheses to this equation we have

$$
\Delta^{\prime}{ }_{M}(\alpha \boldsymbol{u})=\left(\Delta_{P} \alpha\right) \boldsymbol{u} .
$$

This theorem, then, becomes the direct consequence of the formula

$$
\begin{aligned}
\int\left(\Delta_{P} \alpha\right) \boldsymbol{u} d \boldsymbol{\tau}=-\int\left\{\left(\frac{d \alpha}{d P} \boldsymbol{n}\right) \boldsymbol{u}-\left(\alpha \frac{d \boldsymbol{u}}{d P}\right) \boldsymbol{n}\right\} & d \sigma \\
& +\int \alpha\left(\Delta^{\prime} \boldsymbol{u}\right) d \tau .^{*}
\end{aligned}
$$

Another form of this theorem is

$$
\int \Delta^{\prime}{ }_{M}(\alpha \boldsymbol{u}) d \tau=\int\left(\Delta_{P} \alpha\right) \boldsymbol{u} d \tau,
$$

which holds even if the subscript $P$ be replaced by $M$ provided, of course, that $\boldsymbol{u}$ is restricted by the conditions of the theorem.

Theorem 6. If $\alpha \cdot d \beta=d \beta \cdot \alpha$, then

$$
\int \alpha \operatorname{grad} \beta d \tau=-\int \alpha \beta \boldsymbol{n} d \sigma-\int \beta \operatorname{grad} \alpha d \tau .
$$

This theorem is easily proved by using the addition theorem for grad $\alpha \beta$ and by applying the "gradient theorem " $\dagger$

$$
\int \operatorname{grad} \alpha d \tau=-\int \alpha \text { nd } \sigma \text {. }
$$

As a special consequence of this one finds, upon replacing $\beta$ by the scalar $m$, that

$$
\int \alpha \operatorname{grad} m d \tau=-\int m \alpha \mathbf{n} d \sigma-\int m \operatorname{grad} \alpha d \tau .
$$

The following associated theorem may be proved in a manner similar to that just suggested:

Theorem 7. If $\alpha \cdot d \beta=d \beta \cdot \alpha$ then

$$
\int(\operatorname{Rot} \alpha) \beta d \tau=-\int \boldsymbol{n} \wedge \alpha \beta d \sigma-\int(\operatorname{Rot} \beta) \alpha d \tau .
$$

The purpose of the first five theorems is to transfer the derivative operator from the formal product $\alpha \boldsymbol{u}$ to the homography $\alpha$ or to the vector $\boldsymbol{u}$ alone. The need for such transformations arises naturally in certain studies in applied mathematics.

The University of Michigan, October 6, 1915 .

* B. M., 56 [3], third.

$\dagger$ B. M., $55[3]$. 